\title{
Chemotaxonomy and flavonoid diversity of Salvia L. (Lamiaceae) in Iran
}

\author{
Navaz Kharazian ${ }^{1,2}$
}

Received: 26 March, 2013. Accepted: 4 December, 2013

\begin{abstract}
In this study, we evaluated the chemotaxonomic status and chemical diversity of Salvia L. species in Iran using leaf flavonoid profiles. From natural habitats in the country, we collected samples of 14 species of the genus: S. spinosa L.; S. macrosiphon Boiss.; S. reuterana Boiss.; S. sharifii Rech.f. \& Esfand.; S. nemorosa L.; S. virgata Jacq.; S. syriaca L.; S. mirzayanii Rech.f. \& Esfand.; S. atropatana Bunge; S. limbata C. A. Mey; S. sclarea L.; S. ceratophylla L.; S. multicaulis Vahl.; and S. hydrangea Dc. ex Benth. Two-dimensional maps of these species were created with thin-layer chromatography. In order to study the taxonomic position of these species and 37 accessions, cluster analysis was applied. The results of the cluster analysis showed that $S$. spinosa was distinct from S. reuterana. Despite considerable morphological similarity between S. nemorosa and S. virgata, those two species are definitely distinguished. In addition, S. spinosa and S. macrosiphon were roughly grouped, whereas S. ceratophylla and S. multicaulis composed two separate groups. In the 14 species collected, the flavonoids identified were flavones, flavonols, flavanones, isoflavones, dihydroflavonols and chalcones. We found that flavonoids are appropriate indicators to determine the taxonomic position of Salvia species.
\end{abstract}

Key words: thin-layer chromatography, chemical diversity, Salvia, flavonoid, Lamiaceae

\section{Introduction}

The genus Salvia L. belongs to the Mentheae tribe within the Nepetoideae subfamily of the family Lamiaceae. Salvia L. is an important genus, with more than 1000 species worldwide, including 56 species in Iran (Hedge 1982b; Walker et al. 2004). It has a cosmopolitan distribution, occurring in arctic, subarctic, temperate, subtropical and tropical areas, including tropical regions of Iran (Hedge 1982b; Walker et al. 2004). Some of these species are annual, perennial, herbaceous, suffruticose, fruticose and subshrubby (Hedge 1982b; Khan et al. 2002). The main speciation centers of these taxa are considered to be the eastern Mediterranean region; the southwestern, western, eastern and central regions of Asia; Southern Africa; and Central and South America (Hedge 1990; Walker et al. 2004; Kahraman \& Dogan 2010).

It is known that Salvia taxa are used in traditional medicines throughout the world (Anackov et al. 2009). The genus has a wide, cosmopolitan distribution and displays a remarkable range of variation (Walker et al. 2004). Salvia species have been found to have significant biological effects (Sajjadi \& Ghannadi 2005), as well as showing high diversity in their secondary metabolites (Flamini et al. 2007).

According to the taxonomic and morphologic literature, the sections or groups identified in Salvia (Boissier 1879; Hedge 1982b) are not in accordance with each other
(Valant-Vestachera et al. 2003). Having such morphological and genomic variability throughout the world, this genus occupies a significant taxonomic position among the plant biosystematics and taxonomy (Baikova 1996). In addition, there is great similarity in morphological characters and considerable hybridization among some Salvia species; the genus presents high diversity in terms of polyploid levels and karyotypes (Kharazian 2011); and it is a genus of taxonomic, ecological and genomic complexity. Consequently, the species boundaries have been blurred and no satisfactory classification system yet exists (Valant-Vestachera et al. 2003).

Chemotaxonomic studies constitute one of the most important methods of determining the taxonomic positions of taxa. It is now possible to study phenolic profiles of low and high taxonomic levels, even of individual genotypes (Mika et al.2005). Evaluating the patterns of distribution of natural plant products is well established as a major tool for investigating accession structures, species, taxonomic problems and phyletic relationships among genera (Nakiboglu 2002). In addition, flavonoid compounds have been proven to be of chemotaxonomic importance (Fairbbothers et al. 1975; Adzet et al. 1988; Tomas-Barberan \& Wollenweber 1990; Nakiboglu 2002; Valant-Vestachera et al. 2003; Kharazian \& Rahiminejad 2008, 2009). The Salvia genus is a rich source of flavonoid and phenolic acid (Lu \& Foo 2000, 2002; Amiri 2007). Consequently, the chemotaxonomy research

\footnotetext{
${ }^{1}$ Shahrekord University, Faculty of Sciences, Department of Botany, Shahrekord, Iran

${ }^{2}$ Author for correspondence: nkharazian@gmail.com
} 
of Salvia species has focused on their external flavonoid compounds (Adzet et al. 1988; Nakiboglu 2002; Lu \& Foo 2002; Nikolova et al. 2006; Habibvash et al. 2007; Gohari et al. 2011). In addition, the chemotaxonomic status of the Salvia genus has not been exactly determined and needs to be revised in terms of the systematic positions (Nakiboglu 2002). Based on our additional research, using amplified fragment length polymorphism (AFLP) molecular markers, we determined the genetic diversity of the Salvia genus in Iran for the first time (Sajadi et al. 2010). Valant-Vetschera et al. (2003) reported that Salvia species show high chemical diversity and largely aggregated flavone composition.

To our knowledge, there have been no chemotaxonomic studies of the various species of Salvia in Iran. Because Salvia species are distributed in the region, the aim of the present study was to determine the chemotaxonomic status and chemical diversity within the gene pool of the Salvia genus. Since some Salvia species show great interspecific similarity, and considerable morphological variations, we studied three groups of Salvia species: group E-S. spinosa L., S. macrosiphon Boiss., S. reuterana Boiss., S. sharifii Rech.f. \& Esfand., S. nemorosa L., S. virgata Jaq., S. syriaca L. and S. mirzayanii Rech.f. \& Esfand.; group D-S. atropatana Bunge, S. limbata C. A. Mey, S. sclarea L., S. ceratophylla L.; and group B-S. hydrangea Dc. ex Benth. and S. multicaulis Vahl. To our knowledge, this is the first report on the chemotaxonomy and chemical diversity of Salvia species and accessions in Iran.

\section{Material and methods}

\section{Plant material}

The locations of the Salvia species evaluated from collected material $(\mathrm{n}=14)$ and from accessions $(\mathrm{n}=37)$, all collected from natural habitats in Iran, are shown in Tab. 1. Voucher specimens were deposited in the Herbarium of Shahrekord University, in the city of Shahrekord, Iran. For each Salvia species, we evaluated morphological characters such as leaf, bract, calyx, corolla, style and nutlet features (Tab. 2 and 3).

\section{Sample extraction}

The extraction of flavonoids followed the protocol devised by Markham (1982) and Ciesla \& WaksmundzkaHajnos (2010). The flavonoid solution was extracted from air-dried leaves (10.5 g) of 14 Salvia species (Tab. 1) using crude $85 \% \mathrm{MeOH}$ at $60^{\circ} \mathrm{C}$. The solvent was removed from the extract with a rotary evaporator at $70^{\circ} \mathrm{C}$ for total solvent removal and purification of the flavonoids from carotene and chlorophyll was provided using $\mathrm{n}-\mathrm{BuOH}$ and subsequently analyzed by two-dimensional mapping on silica gel 60F $254\left(30 \mathrm{mg}, 67.5 \mathrm{ml} \mathrm{H}_{2} \mathrm{O}\right.$ ) thin-layer chromatography (TLC; $5 \mu \mathrm{m}, 20 \times 20 \mathrm{~cm}$ ). The chromatogram was developed in $\mathrm{BuOH}-\mathrm{C}_{2} \mathrm{H}_{4} \mathrm{O}_{2}-\mathrm{H}_{2} \mathrm{O}$ (BAW 3:1:1) representing an organic system. Spot detection with natural product identifiers (5\% $\mathrm{H}_{2} \mathrm{SO}_{4} / \mathrm{MeOH}$ ) was performed under ultraviolet light at 366 $\mathrm{nm}$ (Nakiboglu 2002). The presence/absence of spots was taken as the character state and was applied in each species. In addition, we studied the relative mobility $\left(\mathrm{R}_{\mathrm{f}}\right.$ migration distance of the bands/distance of the solvent front) for each species (Gulen et al. 2004). In order to show the taxonomic position of these species, we performed a cluster analysis based on Euclidean distances with Ward's method, focusing on the organic phase and presence and absence of flavonoid spots in TLC profiles, with the Statistical Package for the Social Sciences, version 20.0 (SPSS Inc., Chicago, IL, USA). The purification of flavonoid compounds of each species was carried out with a chromatography column $(65 \times 3 \mathrm{~cm})$ with sephadex LH20 (Sephadex with 20\% MeOH; SigmaAldrich, St. Louis, MO, USA) in $100 \mathrm{ml} \mathrm{MeOH}$ solution (with graded solutions of $20 \%, 40 \%, 60 \%, 80 \%$ and $100 \%$ of $\mathrm{MeOH}$ and with acetone) and extracted in fractions (the amount of packing material was $50 \mathrm{ml}$ for each level of $\mathrm{MeOH}$ content $(20 \%, 40 \%, 80 \%, 100 \%)$ and for acetone. The fractions were subjected to one-dimensional mapping on silica gels $(3 \mu \mathrm{m})$. Identification of purified compounds was performed on the basis of their ultraviolet spectra (366 $\mathrm{nm}), \mathrm{MeOH}$ solution and shift reagents such as $\mathrm{AlCl}_{3}, \mathrm{AlCl}_{3} /$ $\mathrm{HCl}, \mathrm{NaOAc}, \mathrm{NaOAc} / \mathrm{H}_{3} \mathrm{BO}_{3}$ and $\mathrm{MeOH}$.

\section{Results}

The two-dimensional flavonoid patterns of crude extract obtained from each Salvia species showed colored spots on chromatography plates. The total numbers of spots obtained for each species and accession were as follows: $S$. spinosa-34, 14 and 9 spots; S. macrosiphon-27, 18 and 14 spots; S. reuterana-29 and 19 spots; S. sharifii-23 and 10 spots; S. nemorosa-28, 12 and 11 spots; S. virgata -16 and 11 spots; S. syriaca-50, 12 and 10 spots; S. mirzayanii-22 and 15 spots; S. multicaulis-53 and 13 Spots; S. hydrangea-57, 20 and 21 spots; S. atropatana-34, 15 and 20 spots; S. limbata-46, 13 and 15 spots; S. ceratophylla-24, 10 and 12 spots; and S. sclarea-40, 10 and 11 spots. The color spots detected in 14 Salvia species were as follows (Tab. 4 and 5): white-yellow, dark yellow, white-blue, orange, fluorescent yellow, brown, pale violet, fluorescent blue, pale yellow, pale blue, pale orange, yellow-blue, dark brown and yellow-orange.

In some of the species studied, we observed color variations and new color spots after the detection of natural products (Tab. 4 and 5): yellow, violet, pale violet, blue, pale blue, orange, pale orange, brown, dark yellow, pale yellow, white-yellow, yellow-blue, white-blue, fluorescent blue and fluorescent yellow. Those color spots were first reported for Salvia species in Iran. The flavonoid classes in the 14 Salvia species evaluated are flavones, isoflavones, flavanones, flavonols, dihydroflavonols and chalcones (Tab. 6). 
Table 1. The locality of Salvia species in natural habitats of Iran.

\begin{tabular}{|c|c|c|}
\hline Species (accession) & Locality & Altitude $(\mathrm{m})$ \\
\hline S. macrosiphon (119) & $\begin{array}{c}\text { Chaharmahal va Bakhtiari - } \\
\text { rousta-e Ilbagi }\end{array}$ & 1787 \\
\hline S. macrosiphon (128) & Isfahan - Hojat abad & 2189 \\
\hline S. macrosiphon (63) & Fars - Marvdasht & 1700 \\
\hline S. spinosa (104) & $\begin{array}{l}\text { Chaharmahal va Bakhtiari - } \\
\text { Sardabe Rostam abad }\end{array}$ & 1860 \\
\hline S. spinosa (111) & Isfahan - Shams abad & 1788 \\
\hline S. spinosa (106) & Tehran - Jajaroud & 1250 \\
\hline S. reuterana (27) & $\begin{array}{l}\text { Chaharmahal va Bakhtiari - } \\
\text { Cheshmeh Sayad }\end{array}$ & 1956 \\
\hline S. reuterana (2) & Isfahan - Semirom & 2105 \\
\hline S. syriaca (36) & Lurestan - Khorramabad & 2008 \\
\hline S. syriaca (98) & $\begin{array}{l}\text { Chaharmahal va Bakhtiari - } \\
30 \mathrm{~km} \text { from Ardal, Amir abad }\end{array}$ & 1987 \\
\hline S. syriaca (37) & Guilan - Deylaman & 1500 \\
\hline S. nemorosa (109) & $\begin{array}{c}\text { Chaharmahal va Bakhtiari - } \\
\text { Gandoman }\end{array}$ & 1867 \\
\hline S. nemorosa (150) & $\begin{array}{l}\text { Chaharmahal va Bakhtiari - } \\
\text { Tang-e sayad, Dasht-e chah }\end{array}$ & 1730 \\
\hline S. nemorosa (129) & Isfahan - Naghane, Semirom & 1864 \\
\hline S. virgata $(78)$ & Isfahan - Sad-e- Zayanderoud & 2310 \\
\hline S. virgata (12) & $\begin{array}{c}\text { Chaharmahal va Bakhtiari - } \\
\text { Dastgerd }\end{array}$ & 1980 \\
\hline S. sharifii (60) & Isfahan - Kolah Ghazi & 1700 \\
\hline S. sharifii (61) & Isfahan - Kolah Ghazi & 1660 \\
\hline S. mirzayanii (110) & Fars - Shiraz & 1700 \\
\hline S. mirzayanii (2) & Fars - Marvdasht & 1850 \\
\hline S. hydrangea (115) & Fars - Abadeh & 1800 \\
\hline S. hydrangea (131) & Isfahan - Semirom & 1791 \\
\hline S. hydrangea (168) & $\begin{array}{c}\text { Chaharmahal va Bakhtiari - } \\
\text { Borujen }\end{array}$ & 2000 \\
\hline S. multicaulis (158) & Isfahan - Semirom, Vanak & 1950 \\
\hline S.multicaulis (166) & $\begin{array}{c}\text { Chaharmahal va Bakhtiari - } \\
\text { Do Polan }\end{array}$ & 1659 \\
\hline S. multicaulis (157) & Ahvaz - Izeh & 1830 \\
\hline S. ceratophylla (138) & $\begin{array}{c}\text { Chaharmahal va Bakhtiari - } \\
\text { Bostan Shir }\end{array}$ & 2120 \\
\hline S. ceratophylla (112) & $\begin{array}{c}\text { Isfahan - } 45 \mathrm{~km} \text { from } \\
\text { Lordegan }\end{array}$ & 1771 \\
\hline S. ceratophylla (137) & Isfahan - Vanak Semirom & 1815 \\
\hline S. sclarea (164) & Isfahan -Daran, Damane & 1856 \\
\hline S. sclarea (163) & $\begin{array}{l}\text { Chaharmahal va Bakhtiari - } \\
\text { Naghan, Firouz abad }\end{array}$ & 1988 \\
\hline S. sclarea (165) & $\begin{array}{c}\text { Kohgilouye va Boyer Ahmad } \\
\text { - Sisakht }\end{array}$ & 1800 \\
\hline S. atropatana (135) & Kurdestan - Marivan & 1820 \\
\hline S. atropatana (134) & Isfahan - Semirom & 1836 \\
\hline S. atropatana (141) & $\begin{array}{c}\text { Chaharmahal va Bakhtiari - } \\
\text { Tange Sayad }\end{array}$ & 1836 \\
\hline S. limbata (124) & $\begin{array}{l}\text { Chaharmahal va Bakhtiari - } \\
\text { Saman, Horeh }\end{array}$ & 2070 \\
\hline S. limbata (123) & $\begin{array}{l}\text { Chaharmahal va Bakhtiari - } \\
\text { Saman, Ben }\end{array}$ & \\
\hline
\end{tabular}

The $R_{f}$ values in organic solvent systems were obtained for each species profile. As can be seen in Tab. 7, the highest $R_{\mathrm{f}}$ in an organic system $\left(\mathrm{R}_{\mathrm{f}}=2.13\right)$ was found for Salvia virgata, whereas the lowest $\left(\mathrm{R}_{\mathrm{f}}=0.01\right)$ was for $S$. atropatana.

In order to determine the taxonomic status of Salvia species, cluster analysis was applied using presence and absence of spots.

The cluster analysis based on the presence and absence of flavonoid spots in the TLC profiles of group E produced two groups (Fig. 1), the first comprising Salvia mirzayanii and $S$. reuterana, and the second comprising two subgroups: S. syriaca, S. nemorosa, S. spinosa and S. macrosiphon; and S. macrosiphon, S. spinosa, S. reuterana, S. virgata, S. sharifii and $S$. nemorosa. Salvia virgata was definitely distinct from $S$. nemorosa. In addition, $S$. sharifii differed from $S$. macrosiphon. Salvia spinosa was clearly separate from $S$. reuterana. A high level of chemical diversity was found in S. spinosa, S. reuterana, S. macrosiphon, S. nemorosa and S. syriaca (Fig. 1 and 2). An additional cluster analysis including four Salvia species with high morphological relationships is shown in Fig. 2. The additional cluster analysis also produced two groups, both of which comprised two subgroups. The first group contained S. sharifii, S. reuterana and S. macrosiphon in one subgroup and S. spinosa in the other. The second group contained S. macrosiphon and $S$. spinosa in one subgroup and S. reuterana in the other. This result showed that $S$. reuterana and S. spinosa were clearly separated. Salvia macrosiphon definitely differed from $S$. sharifii. Furthermore, S. macrosiphon and S. reuterana were closely related, and the former also clustered with S. spinosa (Fig. 2). The cluster analysis of group B and group D also produced two groups, both also comprising two subgroups (Fig. 3). The first group contained S. ceratophylla, S. sclarea and S. atropatana in one subgroup and S. atropatana, S. sclarea and S. multicaulis in the other. The second group contained S. atropatana and S. limbata in one subgroup and S. ceratophylla, S. hydrangea and S. multicaulis in the

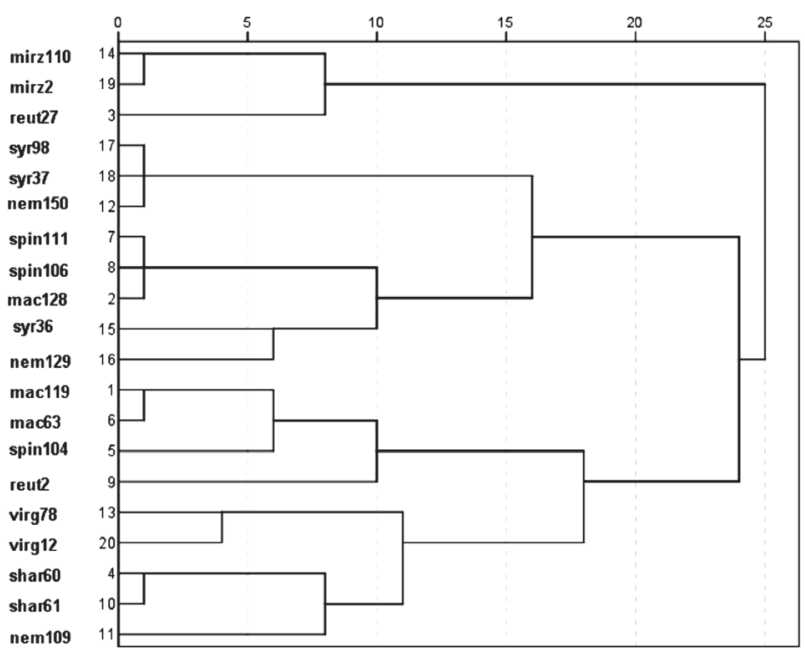

Figure 1. Dendrogram of 20 Salvia accessions belonging to group E. 
Table 2. The morphological characters studied in some Salvia species.

\begin{tabular}{|c|c|c|c|c|c|c|c|c|}
\hline Character & S. macrosiphon & S. reuterana & S. spinosa & S. syriaca & S. nemorosa & S. virgata & S. sharifii & S. mirzayanii \\
\hline Leaf form & $\begin{array}{l}\text { Ovate-oblong, } \\
\text { elliptic, ovate }\end{array}$ & $\begin{array}{l}\text { Ovate, ovate- } \\
\text { oblong }\end{array}$ & $\begin{array}{l}\text { Ovate, broadly } \\
\text { elliptic, ovate- } \\
\text { oblong, broadly } \\
\text { ovate }\end{array}$ & $\begin{array}{l}\text { Ovate, ovate- } \\
\text { oblong, ovate- } \\
\text { lanceolate }\end{array}$ & $\begin{array}{l}\text { Oblong- } \\
\text { lanceolate }\end{array}$ & $\begin{array}{l}\text { Ovate-oblong, } \\
\text { broadly ovate, } \\
\text { oblong }\end{array}$ & Ovate & $\begin{array}{l}\text { Linear, linear- } \\
\text { lanceolate }\end{array}$ \\
\hline $\begin{array}{l}\text { Leaf base } \\
\text { form }\end{array}$ & $\begin{array}{l}\text { Rounded, cordate, } \\
\text { sub-cordate }\end{array}$ & Rounded & $\begin{array}{l}\text { Sub-cordate, } \\
\text { cuneate, rounded, } \\
\text { cordate }\end{array}$ & Cordate & $\begin{array}{l}\text { Cordate, sub- } \\
\text { cordate }\end{array}$ & $\begin{array}{l}\text { Cordate, sub- } \\
\text { cordate }\end{array}$ & Rounded & Cuneate \\
\hline $\begin{array}{l}\text { Leaf margin } \\
\text { form }\end{array}$ & $\begin{array}{l}\text { Sub-entire, } \\
\text { serrate, lobate }\end{array}$ & $\begin{array}{l}\text { Sub-entire, } \\
\text { entire }\end{array}$ & $\begin{array}{l}\text { Entire, erose, sub- } \\
\text { entire, dentate }\end{array}$ & $\begin{array}{l}\text { Erose, } \\
\text { serrulate }\end{array}$ & $\begin{array}{l}\text { Crenate, } \\
\text { serrulate }\end{array}$ & $\begin{array}{l}\text { Erose, sub-entire, } \\
\text { crenulate, serrate }\end{array}$ & $\begin{array}{l}\text { Sub-entire, } \\
\text { serrate }\end{array}$ & Entire \\
\hline $\begin{array}{l}\text { Leaf apex } \\
\text { form }\end{array}$ & $\begin{array}{l}\text { Obtuse, acute, } \\
\text { rounded }\end{array}$ & Obtuse & Acute, obtuse & Acute, obtuse & Acute & $\begin{array}{l}\text { Acute, obtuse, } \\
\text { rounded }\end{array}$ & Acute, obtuse & Acute \\
\hline Bract form & Broadly ovate & Broadly ovate & Broadly ovate & Ovate & Ovate & Ovate & Ovate & Broadly ovate \\
\hline $\begin{array}{l}\text { Bract apex } \\
\text { form }\end{array}$ & Acuminate & Acuminate & $\begin{array}{l}\text { Acuminate- } \\
\text { spinulose }\end{array}$ & Acuminate & Acuminate & Acuminate & Acuminate & Acuminate \\
\hline Bract color & $\begin{array}{l}\text { Green-yellow, } \\
\text { pink }\end{array}$ & Green-yellow & $\begin{array}{l}\text { Green-yellow, } \\
\text { pink, green }\end{array}$ & Green-white & Violet & Green & Green & Violet, white \\
\hline Calyx form & Tubular & $\begin{array}{l}\text { Broadly } \\
\text { tubular }\end{array}$ & Broadly tubular & Tubular & $\begin{array}{l}\text { Tubular- } \\
\text { campanulate }\end{array}$ & $\begin{array}{c}\text { Tubular- } \\
\text { campanulate }\end{array}$ & Tubular & Tubular \\
\hline $\begin{array}{l}\text { Corolla tube } \\
\text { form }\end{array}$ & $\begin{array}{l}\text { Non-invaginated } \\
\text { and non- } \\
\text { squamulose }\end{array}$ & $\begin{array}{l}\text { Non- } \\
\text { invaginated } \\
\text { and non- } \\
\text { squamulose }\end{array}$ & $\begin{array}{l}\text { Non-invaginated } \\
\text { and non- } \\
\text { squamulose }\end{array}$ & $\begin{array}{l}\text { Non- } \\
\text { invaginated } \\
\text { and non- } \\
\text { squamulose }\end{array}$ & $\begin{array}{l}\text { Non- } \\
\text { invaginated } \\
\text { and non- } \\
\text { squamulose }\end{array}$ & $\begin{array}{l}\text { Non-invaginated } \\
\text { and non- } \\
\text { squamulose }\end{array}$ & $\begin{array}{l}\text { Non- } \\
\text { invaginated } \\
\text { and non- } \\
\text { squamulose }\end{array}$ & $\begin{array}{l}\text { Non- } \\
\text { invaginated } \\
\text { and non- } \\
\text { squamulose }\end{array}$ \\
\hline $\begin{array}{l}\text { Style apex } \\
\text { form }\end{array}$ & $\begin{array}{c}\text { Broad } \\
\text { dichotomous }\end{array}$ & $\begin{array}{c}\text { Simple } \\
\text { dichotomous }\end{array}$ & $\begin{array}{l}\text { Simple, broad } \\
\text { dichotomous }\end{array}$ & $\begin{array}{c}\text { Broad } \\
\text { dichotomous }\end{array}$ & $\begin{array}{c}\text { Thin } \\
\text { dichotomous }\end{array}$ & Thin dichotomous & $\begin{array}{c}\text { Broad } \\
\text { dichotomous }\end{array}$ & - \\
\hline Nutlet form & Broad ovoid & Sub-spherical & $\begin{array}{l}\text { Rounded- } \\
\text { trigonous, } \\
\text { spherical }\end{array}$ & $\begin{array}{l}\text { Rounded- } \\
\text { trigonous }\end{array}$ & Ovoid & Ovoid & Ovoid & Ovoid \\
\hline Nutlet color & Light brown & Light brown & $\begin{array}{l}\text { Light brown, dark } \\
\text { brown, light gray }\end{array}$ & Yellow & $\begin{array}{l}\text { Black, dark } \\
\text { brown }\end{array}$ & Black, dark brown & Light brown & Black \\
\hline
\end{tabular}

Table 3. The morphological characters studied in some Salvia species.

\begin{tabular}{|c|c|c|c|c|c|c|}
\hline Character/species & S. multicaulis & S. hydrangea & S. limbata & S. atropatana & S. ceratophylla & S. sclarea \\
\hline Leaf form & $\begin{array}{l}\text { Elliptic, } \\
\text { suborbicular }\end{array}$ & Pinnatisect & $\begin{array}{l}\text { Broad ovate, broad } \\
\text { cordate }\end{array}$ & $\begin{array}{c}\text { Broad elliptic, oblong, } \\
\text { oblanceolate, anguste } \\
\text { elliptic }\end{array}$ & Pinatifida & $\begin{array}{l}\text { Ovate, ovate- } \\
\text { oblong, obovate }\end{array}$ \\
\hline Leaf base form & Cordate, oblique & Narrow & Cordate & $\begin{array}{l}\text { Oblique, cuneate, } \\
\text { lobed, erose, subentire }\end{array}$ & Narrow & Cordate \\
\hline Leaf margin form & Crenulate, rugose & Entire & Erose & $\begin{array}{c}\text { Sinuate, crenate, } \\
\text { dentate }\end{array}$ & Lobed & $\begin{array}{c}\text { Crenate, erose, } \\
\text { lobed }\end{array}$ \\
\hline Leaf apex form & Obtuse & Acute & Obtuse, acute & Obtuse & Obtuse & Acute, obtuse \\
\hline Bract form & Broadly ovate, lobed & Ovate & Broadly ovate, lobed & Broadly ovate & Broadly ovate & Broadly ovate \\
\hline Bract apex form & Acuminate & Acuminate & Acuminate & Acuminate & Cuspidate & Acuminate \\
\hline Bract color & $\begin{array}{l}\text { Green, pale violet, } \\
\text { Pale pink }\end{array}$ & Green & Green & $\begin{array}{l}\text { Green-yellow, green- } \\
\text { pink }\end{array}$ & Green & White-green, pink \\
\hline Calyx form & Late campanulate & $\begin{array}{l}\text { Campanulate- } \\
\text { infundibuliformis }\end{array}$ & $\begin{array}{l}\text { Tubular- } \\
\text { campanulate, } \\
\text { campanulate }\end{array}$ & Campanulate & Ovate-campanulate & Ovate-campanulate \\
\hline Corolla tube form & Rectus, annulate & $\begin{array}{l}\text { Incompletely annulate } \\
\text { or exannulate }\end{array}$ & $\begin{array}{l}\text { Ventricose, } \\
\text { squamulose }\end{array}$ & $\begin{array}{l}\text { Ventricose, } \\
\text { Squamulose }\end{array}$ & $\begin{array}{l}\text { Ventricose, } \\
\text { squamulose }\end{array}$ & $\begin{array}{l}\text { Ventricose, } \\
\text { squamulose }\end{array}$ \\
\hline Style apex form & $\begin{array}{l}\text { Broad dichotomous, } \\
\text { simple }\end{array}$ & Simple dichotomous & Broad dichotomous & Broad dichotomous & Broad dichotomous & $\begin{array}{l}\text { Broad dichotomous, } \\
\text { simple dichotomous }\end{array}$ \\
\hline Nutlet form & $\begin{array}{l}\text { Ovoid, rounded- } \\
\text { trigonous }\end{array}$ & $\begin{array}{l}\text { Subspherical, } \\
\text { rounded-trigonous }\end{array}$ & Ovoid, spherical & Spherical, ovoid & Spherical & $\begin{array}{l}\text { Rounded, rounded- } \\
\text { trigonous }\end{array}$ \\
\hline Nutlet color & $\begin{array}{l}\text { Black, brown, pale } \\
\text { brown, creamy }\end{array}$ & Pale brown & Pale brown & Yellow-cream, brown & Black, violet & Black, pale brown \\
\hline
\end{tabular}


Table 4. Presence and absence of each spot in Salvia accessions before and after detection of natural products.

\begin{tabular}{|c|c|c|c|c|c|c|c|c|c|c|c|c|c|}
\hline Species & 1 & 2 & 3 & 4 & 5 & 6 & 7 & 8 & 9 & 10 & 11 & 12 & 13 \\
\hline S. macrosiphon (119) &,$++\mathrm{a}$ & + &,$++\mathrm{a}$ &,$++\mathrm{a}$ & - & - &,$-+\mathrm{a}$ &,$-+\mathrm{a}$ & - & - & - & - & - \\
\hline S. macrosiphon (128) & - & - & - &,$++\mathrm{a}$ & - & + & - & - &,$++\mathrm{a}$ & - & - & - & + \\
\hline S. macrosiphon (63) & - & - & - & + & - &,$++\mathrm{a}$ & - & - & - & - & - & - & + \\
\hline S. spinosa (104) &,$++\mathrm{a}$ & - & + & + & - & + & + &,$++\mathrm{a}$ & + & + & - & - & \\
\hline S. spinosa (111) & - & - & + & + & - & - & - & - & - & - & - & - &,$++\mathrm{a}$ \\
\hline S. spinosa (106) & - & - & + & + & - & - & - & - & - & - & - & & + \\
\hline S. reuterana (27) &,$++\mathrm{a}$ & - &,$++\mathrm{a}$ & - & + & - & - &,$-+\mathrm{a}$ & - &,$-+\mathrm{a}$ & $+\mathrm{a}$ & - & - \\
\hline S. reuterana (2) & + & - & - & + & - &,$++\mathrm{a}$ & + & - & - & - & - & - & - \\
\hline S. syriaca (36) &,$++\mathrm{a}$ & - &,$++\mathrm{a}$ & + & - & + & + & + &,$-+\mathrm{a}$ & + & - & - & \\
\hline S. syriaca (98) & - & + & + & - & - & - & - & - & - & - & - & - & - \\
\hline S. syriaca (37) & - & - & + & - & - & - & - & - & - & - & - & - & - \\
\hline S. nemorosa (109) & + & - & + & + & + & + & - & - & - & - & - & $+\mathrm{a}$ & \\
\hline S. nemorosa (150) & - & - & + & - & - & - & - & - & - & - & - &,$++\mathrm{a}$ & - \\
\hline S. nemorosa (129) & + & - & - & - & - & - & - & - & - & - & - & + & - \\
\hline S. virgata (78) & + &,$-+\mathrm{a}$ &,$++\mathrm{a}$ & + & - & - & - &,$-+\mathrm{a}$ & - & - & - & $+\mathrm{a}$ & - \\
\hline S. virgata (12) & + & - & - & + & - & - & - & - & - & - & - & - & - \\
\hline S. sharifii (60) & + &,$++a$ &,$++\mathrm{a}$ & + & - & + & - & - & - & - & - & $+a$ & - \\
\hline S. sharifii (61) & + & - & + & + & - & + & - & - & - & - & - & - & - \\
\hline S. mirzayanii (110) & + & - & + & - & - & - & - & + & - &,$-+a$ & - & - & - \\
\hline S. mirzayanii (2) & + & - & + & + & - & - & - & - & - & - & - & - & - \\
\hline
\end{tabular}

a - the spots after detection of natural product. 1 - yellow, 2 - white-yellow, 3 - blue, 4 - violet, 5 - dark yellow, 6 - white-blue, 7 - orange, 8 - fluorescent yellow, 9 - brown, 10 - fluorescent blue, 11 - pale yellow, 12 - pale blue, 13 - pale violet.

Table 5. Presence and absence of each spot in Salvia accessions before and after detection of natural products.

\begin{tabular}{|c|c|c|c|c|c|c|c|c|c|c|c|c|c|}
\hline Species & 1 & 2 & 3 & 4 & 5 & 6 & 7 & 8 & 9 & 10 & 11 & 12 & 13 \\
\hline S. hydrangea (115) & + & + &,$++\mathrm{a}$ & + & + & $+a$ & - & + & - & $+\mathrm{a}$ & - & - &,$++\mathrm{a}$ \\
\hline S. hydrangea (131) & + & - & + & + & - & - & - & - & - & - & - & - & \\
\hline S. hydrangea (168) & + & - & + & - & - & - & - & - & - & - & - & - & \\
\hline S. multicaulis (166) &,$++\mathrm{a}$ & $+\mathrm{a}$ &,$++\mathrm{a}$ & - & - & + & - & + & - & - & + & - & \\
\hline S. multicaulis (157) & + & - & + & - & + & - & - & - & + & - & - & - & \\
\hline S. multicaulis (158) & + & - & - & - & + & - & - & - & + & - & - & - & \\
\hline S. ceratophylla (138) &,$++\mathrm{a}$ & - & $+a$ & - & - & + & - & - & - & - & - & - & \\
\hline S. ceratophylla (112) & + & - & - & + & - & - & - & - & - & & - & - & \\
\hline S. ceratophylla (137) & + & - & + & + & - & - & - & - & + & - & - & - & \\
\hline S. sclarea (164) &,$++\mathrm{a}$ &,$++\mathrm{a}$ & + & $+\mathrm{a}$ & - & - & + & + & + & - & - & - & \\
\hline S. sclarea (163) & + & - & + & - & + & - & - & - & + & - & - & - & \\
\hline S. sclarea (165) & - & - & + & + & + & - & - & - & + & - & - & - & \\
\hline S. atropatana (135) &,$++\mathrm{a}$ &,$++\mathrm{a}$ &,$++\mathrm{a}$ & &,$++\mathrm{a}$ & - & - & - & - & - & - & - & \\
\hline S. atropatana (134) & + & - & + & + & + & - & - & - & + & - & - & - & \\
\hline S. atropatana (141) & + & - & + & + & - & - & - & - & + & - & - & - & \\
\hline S. limbata (124) & + &,$++\mathrm{a}$ &,$++\mathrm{a}$ & - & - & $+a$ &,$++\mathrm{a}$ & - & - & - & - & + & \\
\hline S. limbata (123) & - & + & + & + & - & - & + & - & + & - & - & - & \\
\hline
\end{tabular}

a - the spots after detection of natural product. 1 - yellow; 2 - violet; 3 - blue; 4 - orange; 5 - brown; 6 - dark yellow; 7 - fluorescent yellow; 8 - fluorescent blue; 9 - white-blue; 10 - pale orange; 11 - dark brown; 12 - yellow-orange; 13 - yellow-blue. 
Table 6. Flavonoid classes in Salvia accessions.

\begin{tabular}{|c|c|}
\hline Species & Flavonoid class \\
\hline S. hydrangea & $\begin{array}{l}\text { Flavones, flavanones, flavonols, isoflavones, } \\
\text { dihydroflavonols }\end{array}$ \\
\hline S. multicaulis & $\begin{array}{l}\text { Flavones, flavanones, flavonols, isoflavones, } \\
\text { dihydroflavonols, chalcones }\end{array}$ \\
\hline S. ceratophylla & Flavones, flavanones, flavonols,, isoflavones \\
\hline S. sclarea & $\begin{array}{l}\text { Flavones, flavanones, flavonols, isoflavones, } \\
\text { dihydroflavonols, chalcones }\end{array}$ \\
\hline S. atropatana & $\begin{array}{l}\text { Flavones, flavanones, flavonols, isoflavones, } \\
\text { dihydroflavonols, chalcones }\end{array}$ \\
\hline S. limbata & $\begin{array}{l}\text { Flavones, flavanones, flavonols,, isoflavones, } \\
\text { chalcones }\end{array}$ \\
\hline S. macrosiphon & Flavones, flavonols, flavanones \\
\hline S. spinosa & Flavones, flavanones \\
\hline S. reuterana & Flavones, flavanones, flavonols \\
\hline S. syriaca & Flavones, flavanones \\
\hline S. nemorosa & Flavones, isoflavones \\
\hline S. virgata & Flavones, flavonols, isoflavones \\
\hline S. sharifii & Flavones, flavonols, flavanones, chalcones \\
\hline S. mirzayanii & Isoflavones \\
\hline
\end{tabular}

other (Fig. 3). In these results, most of the species displayed chemical diversity. Moreover, S. multicaulis and S. ceratophylla comprised two separate groups.

Morphological characters-leaf form, leaf margin, leaf base form, leaf apex form, bract apex form, bract color, calyx form, corolla tube form, style apex form, nutlet form and nutlet color-were investigated in 14 Salvia species. As can be seen in Tab. 2 and 3, leaf features, bract apex form, calyx form, style apex form, nutlet form and nutlet color were found to be appropriate morphological characters to differentiate among those 14 Salvia species (Kharazian 2012b). Salvia spinosa was distinguished from S. reuterana by the bract apex form, bract color, leaf margin, leaf base form and nutlet features. Salvia nemorosa clearly differed from S. virgata in leaf form, leaf margin form, leaf apex form and bract color. In addition, S. sharifii and S. macrosiphon differed in leaf form, leaf base form, leaf margin and bract form (Tab. 2). It can be concluded that these morphological characters are diagnostic features (Kharazian 2012b).

The taxonomic positions of the 37 Salvia accessions and 14 Salvia species (group B, D and E) were determined using cluster analysis and identification of spots. The cluster analysis produced two groups, each comprising two subgroups (Fig. 4). The first group contained S. mirzayanii, S. reuterana, S. syriaca, S. hydrangea, S. ceratophylla, S. limbata, S. atropatana and S. multicaulis in one subgroup and S. ceratophylla, S. sclarea, S. atropatana and S. multicaulis in the other. The second group contained $S$. nemorosa, S. sharifii and S. virgata in one subgroup and S. spinosa and $S$. macrosiphon in the other. Among the accessions, the main distinction was between $S$. nemorosa and S. virgata. Salvia spinosa was clearly distinct from $S$. reuterana. In addition, S. spinosa accessions were grouped with S. macrosiphon accessions. Salvia ceratophylla clustered with S. hydrangea, S. sclarea and S. atropatana. Salvia limbata was grouped only with S. atropatana. Salvia atropatana, S. multicaulis, S. hydrangea, S. syriaca, S. nemorosa, S. macrosiphon and S. spinosa accessions displayed high chemical diversity (Fig. 4).

Based on the patterns of flavonoid variation (Tab. 8 and 9), we observed B-ring ortho-dihydroxylation in Salvia spinosa,

Table 7. Relative mobility values for Salvia species in the organic solvent system.

\begin{tabular}{lccc}
\hline \multirow{2}{*}{ Species } & & $\mathrm{R}_{\mathrm{f}}$ value & Mean \\
\cline { 2 - 4 } S. hydrangea & Minimum & Maximum & 0.88 \\
S. multicaulis. & 0.13 & 1.39 & 0.87 \\
S. ceratophylla & 0.13 & 1.48 & 1.05 \\
S. sclarea & 0.11 & 1.54 & 0.84 \\
S. atropatana & 0.33 & 1.34 & 0.52 \\
S. limbata & 0.01 & 0.94 & 0.73 \\
S. macrosiphon & 0.31 & 1.31 & 0.61 \\
S. spinosa & 0.16 & 1 & 0.77 \\
S. reuterana & 0.15 & 1.19 & 0.75 \\
S. syriaca & 0.24 & 1.37 & 0.78 \\
S. nemorosa & 0.42 & 1.08 & 0.71 \\
S. virgata & 0.11 & 1.25 & 1.39 \\
S. sharifii & 0.69 & 2.13 & 1.03 \\
S. mirzayanii & 0.59 & 1.29 & 0.77 \\
\hline
\end{tabular}

$R_{f}$ - relative mobility (migration distance of the bands/distance of the solvent front). 


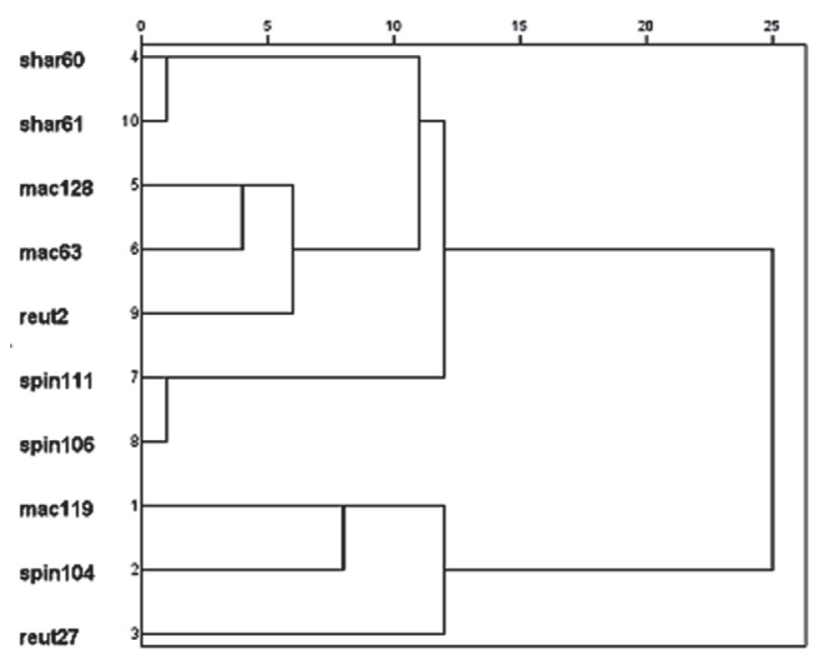

Figure 2. Dendrogram of 10 Salvia accessions of Salvia spinosa, S. reuterana, S. macrosiphon and S. sharifii.

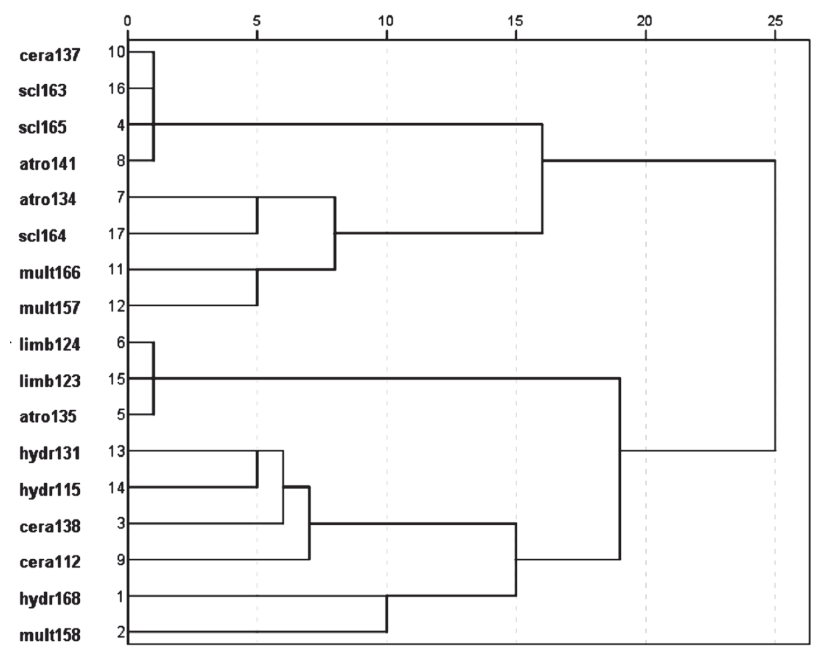

Figure 3. Dendrogram of 17 Salvia accessions belonging to group B and group D.

S. macrosiphon, S. reuterana, S. syriaca, S. mirzayanii, S. nemorosa, S. ceratophylla, S. sclarea and S. limbata, whereas we observed A-ring ortho-dihydroxylation in $S$. spinosa, S. reuterana, S. mirzayanii, S. syriaca, S. nemorosa, S. hydrangea, S. multicaulis, S. sclarea, S. atropatana and S. limbata. In most of the Salvia species, there was a tendency toward 3-hydroxylation, 5-hydroxylation, 7-hydroxylation, 2'-hydroxylation, 3'-hydroxylation, 4'-hydroxylation and 4'-methoxylation. In some species, 2-hydroxylation, 4-hydroxylation, 6-hydroxylation, 8-hydroxylation, 5'-hydroxylation, 5-methoxylation, 6-methoxylation, 7-methoxylation, 8-methoxylation, 2'-methoxylation and 3'-methoxylation were present (Tab. 8 and 9). We also observed other substitutions (Tab. 8 and 9), including 7-o-rhamnoglucosylation; 3-o-glucosylation; 5-o-glucosylation; 7-o-glucosylation; 7-orhamnosylation; 3-o-rhamnuogalactosylation ; 7-o-glucuronosylation; 3-o-rhamnosylation; 3-o-galactosylation; 8-c-

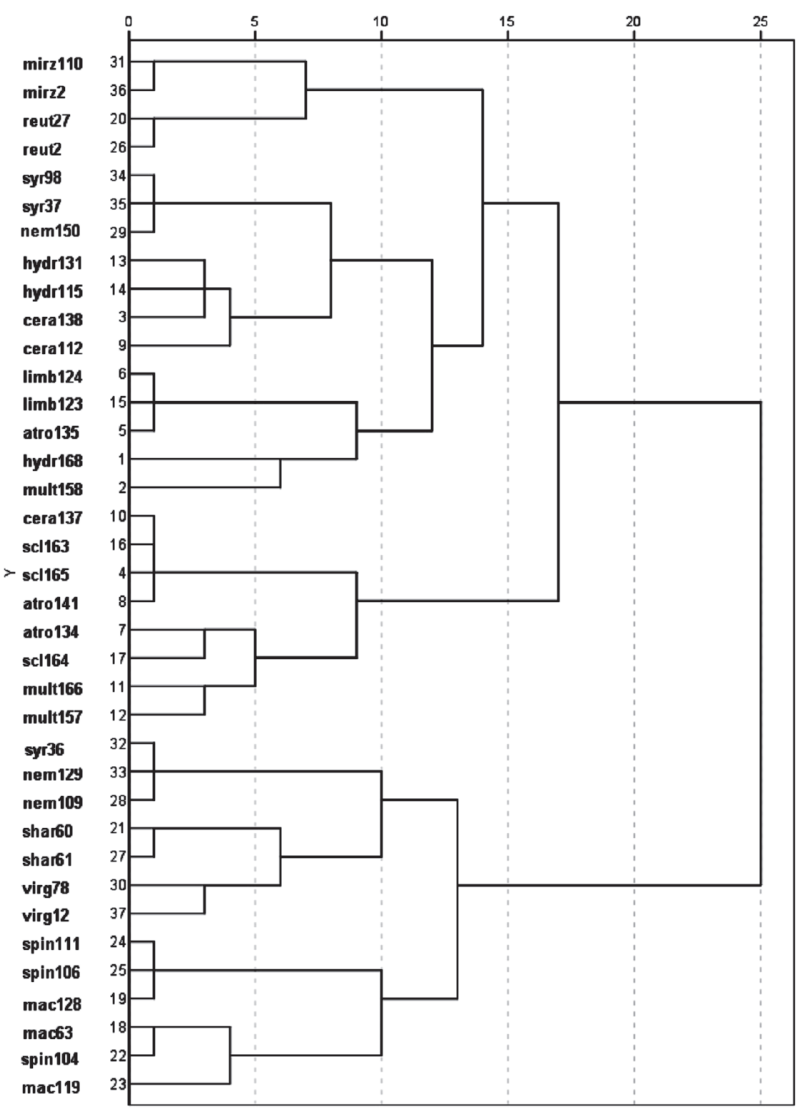

Figure 4. Dendrogram of 37 Salvia accessions belonging to group B, group $\mathrm{D}$ and group $\mathrm{E}$.

rhamnoglucosylation; 8-c-glucosylation; 7-o-rutinosylation; 6-c-glucosylation; 3-, 3'- and 4'-methylene dioxidization; 3-o- $\beta$-glucopyranosylation; and 2-carboxylation. This is the first report of flavonoid variations for Salvia species in Iran.

\section{Discussion}

According to the taxonomic literature, Salvia species are divided into five groups by leaf form, stamen type, corolla tube and calyx form (Hedge 1982b). Salvia syriaca, S. nemorosa, S. reuterana, S. macrosiphon, S. spinosa, S. virgata, S. sharifii and S. mirzayanii constitute a group in which the corolla tube is not invaginated or squamulose and the inside of the corolla tube is imperfectly or perfectly annulated (Hedge 1982b). Morphologically, Salvia reuterana, S. spinosa and S. macrosiphon are closely related. In the present study, the number of spots differ among $S$. reuterana, S. spinosa and S. macrosiphon (29 and 19;34, 14 and 9; and 27, 18 and 14 spots, respectively). In terms of the $\mathrm{R}_{\mathrm{f}}$ values in the organic phase, $S$. spinosa differed from the two other species, which is in agreement with Sajadi et al. (2010). In addition, in the TLC profile of S. spinosa, white-blue and brown spots were observed. Salvia spinosa is a polymorphic taxon with high morphological variability 
Table 8. Flavonoid variation patterns in Salvia species.

\begin{tabular}{|c|c|c|c|c|c|c|c|c|}
\hline Variation pattern & $\mathrm{sp}$ & mac & reut & syr & nem & virg & shar & mirz \\
\hline A-ring ortho-dihydroxylation & + & - & + & + & + & - & - & + \\
\hline B-ring ortho-dihydroxylation & + & + & + & + & + & - & - & + \\
\hline 2-hydroxylation & - & + & - & - & + & - & + & - \\
\hline 3-hydroxylation & + & + & - & - & + & + & + & + \\
\hline 5-hydroxylation & + & + & + & + & + & + & + & + \\
\hline 6-hydroxylation & + & - & + & - & + & - & - & + \\
\hline 7-hydroxylation & + & + & + & + & + & + & + & + \\
\hline 8-hydroxylation & - & + & - & + & + & + & + & + \\
\hline 2'-hydroxylation & - & + & + & - & + & - & + & + \\
\hline 3'-hydroxylation & + & + & + & + & + & + & + & + \\
\hline 4'-hydroxylation & + & + & + & + & + & + & + & + \\
\hline 5'-hydroxylation & - & - & - & - & + & - & - & - \\
\hline 5-methoxylation & - & + & + & - & + & - & - & - \\
\hline 6-methoxylation & - & + & + & - & + & + & + & + \\
\hline 7-methoxylation & - & + & + & + & + & + & + & + \\
\hline 8-methoxylation & - & - & - & - & - & - & - & + \\
\hline 2'-methoxylation & + & - & - & - & - & - & - & - \\
\hline 3'-methoxylation & - & - & - & - & + & + & - & + \\
\hline 4'-methoxylation & + & + & + & + & + & + & + & + \\
\hline 7-o-rhamnoglucosylation & + & - & + & - & + & + & - & - \\
\hline 3'-methylene dioxidization & - & - & - & - & - & + & - & + \\
\hline 4'-methylene dioxidization & - & - & - & - & - & + & - & + \\
\hline 3-o-glucosylation & - & + & + & - & - & - & + & + \\
\hline 5-o-glucosylation & - & - & - & + & + & - & - & - \\
\hline 7-o-glucosylation & - & - & - & + & - & + & - & + \\
\hline 8-c-glucosylation & - & - & - & - & - & + & - & - \\
\hline 6-c-glucosylation & - & - & - & + & - & - & - & + \\
\hline 7-o-rhamnosylation & - & - & + & - & + & - & - & - \\
\hline 3-o-rhamnosylation & - & - & - & - & + & - & - & - \\
\hline 3-o-rhamnogalactosylation & - & - & + & - & - & - & - & - \\
\hline 7-o-glucuronosylation & - & - & + & + & - & - & - & - \\
\hline 3-o-galactosylation & - & - & - & - & + & - & - & - \\
\hline 8-c-rhamnoglucosylation & - & - & - & - & + & - & - & - \\
\hline 7-o-rutinosylation & - & - & - & - & - & + & - & - \\
\hline 2-carboxylation & + & - & + & - & - & - & - & - \\
\hline
\end{tabular}

sp - Salvia spinosa; mac - S. macrosiphon; reut - S. reuterana; syr - S. syriaca; nem - S. nemorosa; virg - S. virgata; shar - S. sharifii; mirz - S. mirzayanii.

(Kharazian 2009; 2012b). Furthermore, some of the S. reuterana accessions from the Azerbaijan province are similar to S. spinosa in term of the calyx in the fruit (Hedge 1982b). Salvia spinosa includes morphological characters that are not easily distinguishable from those of $S$. reuterana. In the present study, the flavonoid profiles, flavonoid types and morphological features were found to be appropriate markers to discriminate the two species. The main morphological characters differentiating S. spinosa from S. reuterana are the bract features (Kharazian 2009). In the present study, $S$. reuterana was distinguished by its flavonol compounds. Conversely, some of the S. spinosa accessions from Turkey are similar to those of S. macrosiphon from Iran and Afghanistan (Hedge 1982a; Kahraman et al. 2009), which are closely grouped using flavonoid profiles. Kharazian (2012b) also showed that $S$. spinosa and S. macrosiphon were closely related morphologically but differs in diagnostic characters such as the form of the leaves and calyx. 
Table 9. Flavonoid variation patterns in Salvia species.

\begin{tabular}{|c|c|c|c|c|c|c|}
\hline Variation pattern & hydr & mult & cera & $\mathrm{scl}$ & atro & $\operatorname{limb}$ \\
\hline A-ring ortho-dihydroxylation & + & + & - & + & + & + \\
\hline B-ring ortho-dihydroxylation & - & - & + & + & - & + \\
\hline 2-hydroxylation & - & - & - & - & - & + \\
\hline 3-hydroxylation & + & + & + & + & + & + \\
\hline 4-hydroxylation & - & + & - & + & + & - \\
\hline 5-hydroxylation & + & + & + & + & + & + \\
\hline 6-hydroxylation & + & + & - & + & - & + \\
\hline 7-hydroxylation & + & + & + & + & + & + \\
\hline 8-hydroxylation & + & + & - & - & - & + \\
\hline 2'-hydroxylation & + & + & + & + & + & + \\
\hline 3'-hydroxylation & + & + & + & + & + & + \\
\hline 4'-hydroxylation & + & + & + & + & + & + \\
\hline 5'-hydroxylation & + & - & - & - & - & - \\
\hline 6-methoxylation & + & + & + & + & + & + \\
\hline 7-methoxylation & - & + & + & - & - & + \\
\hline 8-methoxylation & + & + & + & + & + & + \\
\hline 3'-methoxylation & + & + & + & - & + & + \\
\hline 4'-methoxylation & + & + & + & + & + & + \\
\hline 7-o-rhamnoglucosylation & - & + & - & - & - & + \\
\hline 3-methylene dioxidization & - & - & + & + & - & + \\
\hline 3'-methylene dioxidization & + & + & + & - & + & + \\
\hline 5-o-glucosylation & - & - & - & - & - & + \\
\hline 8-c-glucosylation & - & - & + & - & - & - \\
\hline 6-c-glucosylation & - & - & + & - & - & - \\
\hline 3-o-glucosylation & - & + & - & + & + & - \\
\hline 7-o-glucosylation & - & - & - & - & + & + \\
\hline 7-o-glucuronosylation & + & + & - & + & + & + \\
\hline 3-o-galactosylation & - & + & - & - & - & - \\
\hline 3-o- $\beta$-glucopyranosylation & - & - & + & - & - & + \\
\hline
\end{tabular}

hydr - Salvia hydrangea; mult - S. multicaulis; cera - S. ceratophylla; scl - S. sclarea; atro - S. atropatana; limb - S. limbata.

In our cluster analysis, Salvia reuterana and S. macrosiphon were in separate groups. This differentiation was not observed by Sajadi et al. (2010). Salimpour et al. (2011), using essential oil composition, found that these two species were different, which is in agreement with our results. It can be concluded that environmental conditions and forms of secondary metabolites contribute to the different results obtained with molecular markers (Maksimovic et al. 2007).

In the cluster analysis, Salvia syriaca was grouped with S. nemorosa. Nevertheless, one S. syriaca accession was quite different from the other species in term of the number of color spots. In the flora of Turkey and Russia, S. syriaca has been mentioned in a separate group (Pobedimova 1954; Hedge 1982a). In our results, using the AFLP molecular marker, Sajadi et al. (2010) also reported that S. syriaca accessions were grouped with $S$. nemorosa, which is in agreement with Bagcia et al. (2004), Goren et al. (2006) and Kharazian (2012b). It can be concluded that $S$. syriaca accessions showed high chemical diversity. In addition, our results are in accordance with the findings of Bagcia et al. (2004), Goren et al. (2006) and Habibvash et al. (2007), who used fatty acid compositions and phenolic compounds. Kharazian (2012b) and Sajadi et al. (2010) showed high morphological and molecular diversity in S. syriaca accessions.

On the basis of our data, Salvia nemorosa was grouped with other species, which is correlated with the hybridization of S. nemorosa with different species (Hedge 1982a, 1982b; Sajadi et al. 2010). Janicsak et al. (2006) reported the variability in oleanolic and ursolic acid contents among subspecies of $S$. nemorosa. In addition, S. nemorosa is clearly separate from S. virgata, which is based on Sajadi et al. (2010). Furthermore, in the flora of Russia, these two species were 
mentioned in two separate series (Pobedimova 1954). Notably, these two species have a high morphological similarity, which makes it difficult to separate the two. As was observed in the present study, the two species are distinguished by bract color and leaf features (Kharazian 2012b). Evidently, in the organic phase, S. virgata is a separate species, which is based on Goren et al. (2006). Evaluating nutlet anatomy, Habibvash \& Rajamand (2007) reported that S. virgata and $S$. nemorosa were distinct. Moreover, nutlet morphology in S. virgata showed that the apomorphic characteristic of this species distinguishes it from all other Salvia taxa (Ozkan et al. 2009). Tosun et al. (2009) also mentioned that these two species showed significant differences in antioxidant activity and total phenolic compounds. It can be concluded that the organic phase is appropriate and can be well documented. In our study, it seemed that flavonol compounds differentiated these two species. The presence of fluorescent yellow in $S$. virgata is supported by the chemotaxonomy results obtained by Nakiboglu (2002). Notably, Nakiboglu (2002) reported only four spots for this species, which is not in accordance with our findings (16 and 11 spots).

In the cluster analysis, Salvia sharifii and S. macrosiphon were grouped separately (Sajadi et al. 2010). Some of the $S$. sharifii accessions from the southern and southeastern regions of Iran have been associated with S. macrosiphon (Hedge 1982b), which is not supported our results. It seems that the bract form and leaf features are one of the main morphological characters for separating these two species (Kharazian 2012b). In addition, the two species differed in terms of the flavonoid classes presented.

The results of our cluster analysis in 10 Salvia accessions, including S. macrosiphon, S. reuterana, S. spinosa and S. sharifii, show that these species are clearly related, which is in agreement with the results of Kharazian (2009) and Sajadi et al. (2010). It can be concluded that the presence and absence of flavonoid spots was a significant factor in determining the taxonomic status of Salvia species.

The cluster analyses of Salvia accessions showed high chemical diversity in S. spinosa, S. syriaca, S. reuterana, S. nemorosa and $S$. macrosiphon. Owing to the hybridization and introgression of $S$. macrosiphon with other species, such as S. moorcroftiana Wall. Ex Benth. and S. reuterana, there are also patterns of variation in S. macrosiphon (Hedge 1982a; 1990). Based on the results obtained by Kharazian (2009; 2012b), most of those variations were in leaf indumentum, leaf form, leaf base, leaf margin, bract indumentum, corolla color, corolla tube length, calyx length, calyx apex form and inflorescence indumentum. In addition, Kharazian (2012b) mentioned that the morphological variations in S. spinosa accessions were in leaf form, leaf margin form, leaf indumentum, stem indumentum, inflorescence indumentum, bract indumentum, bract dimension, bract color, calyx indumentum, calyx dimension, corolla indumentum and corolla length. Conversely, S. virgata, S. sharifii and S. mirzayanii accessions displayed no chemical diversity.
It has been reported that the variability in flavonoid patterns is influenced by ecological conditions (Tomas-Barberan \& Wollenweber 1990).

Salvia multicaulis, S. hydrangea and S. ceratophylla were placed in a group of species with pinnatisect leaves, and $S$. multicaulis also belongs to a group in which the anthers have lower thecae and the calyx in the fruit is expanded and membranous-reticulate. Salvia atropatana, S. ceratophylla, S. sclarea and S. limbata were included in a group of species with invaginated, squamulose corolla tubes, the inside of which is glabrous. In the cluster analysis of these groups, $S$. multicaulis was included in two groups and was clustered with S. hydrangea. Kharazian (2012b) reported that the morphological variations in S. multicaulis are generally related to the indumentum of the stem and leaf; petiole; calyx and inflorescence axis; leaf form; calyx apex; calyx color; and bract form. It appears that the morphological variations in S. multicaulis are closely related to the varieties, forms or polymorphism characters of the species. Sajadi et al. (2010) also reported molecular variations among the $S$. multicaulis accessions. Salvia ceratophylla accessions were grouped with those of S. sclarea, S. atropatana and S. hydrangea. Sajadi et al. (2010) and Kharazian (2012b) reported that S. ceratophylla accessions were mostly grouped with S. sclarea. In addition, Habibvash et al. (2007) reported that S. ceratophylla and S. sclarea are similar in terms of their contents of linolenic and arachidic acid, which is in keeping with our results. In the present study, S. ceratophylla was also included in two groups. We found that S. atropatana was clustered with S. sclarea, S. limbata and S. ceratophylla. Using fatty acid, Habibvash et al. (2007) reported this relationship in S. limbata, S. sclarea and S. ceratophylla. According to Kharazian (2012a; 2012b), S. atropatana displays considerable variation in morphological characters such as leaf form, leaf margin form, leaf indumentum, bract indumentum, calyx indumentum, corolla indumentum and style length. We also found flavonoid variations in S. atropatana. Salvia limbata accessions were grouped with S. atropatana, which is in agreement with Sajadi et al. (2010) and Kharazian (2012b). Notably, the two species presented similar classes of flavonoids. We observed flavonoid variations in all of the Salvia species belonging to group D. It has been noted that some accessions of $S$. sclarea were included in different groups, which is probably due to morphological variations or ecological adaptations (Kharazian 2012b; Ozdemir \& Senel 1999). It seems that these taxonomic differentiations were due to polymorphism in the morphological characters, hybridization between species and geographical distribution. Salvia sclarea shows variability in terms of the bract color, bract length, color of the upper lip of the corolla, leaf form and leaf margin (Kharazian 2012b). It seems that flavonoid diversity and its variation is related to morphological variability and to the geographical conditions in Iran.

Flavones, flavanones and flavonols were common in all of the group studied. The species belonging to group $\mathrm{E}$ might be separated from two other group using dihydrofla- 
vonols. In addition, most of the chalcones and isoflavones were observed in group B and D. It might be concluded that, based on the flavonoid compounds, group B and D were closely related.

On the basis of spot colors, some of the species evaluated have been found to contain isoflavones and flavonols (Tomas-Barberan \& Wollenweber 1990; Lu \& Foo 2002; Amiri 2007). It seems that these compounds were flavones 7-o-rahmnoglucoside; flavones 5-o-glycosides; $5-\mathrm{OH}$ flavanones, flavone 3-o-glucoside, flavones, flavonol, 5-hydroxylflavonol, isoflavone, flavanone, 5-hydroxylflavanone and dihydroflavonol. In the above cases, the variations in flavonoid patterns of hydroxylation, methoxylation, $o$ glucosylation, $o$-glycosylation and $o$-rhamnoglucosylation, $o$-rhamnosylation, $o$-rhamnogalactosylation, $c$-rhamnoglucosylation, $o$-rutinosylation, $o$-galactosylation, $o$-glucuronosylation, $c$-glucosylation, methylene dioxidization and $\beta$-glucopyranosylation occurred in these species which is roughly in accordance with Tomas-Barberan \& Wollenweber (1990) and Lu \& Foo (2002). Consequently, the widest range of flavonoid variations was found in Grex E. It seems that the variations of flavonoid patterns in 14 Salvia species could accurately resolve the taxonomic status.

\section{Conclusion}

In conclusion, we can state that flavonoid profiles, as identified through chemotaxonomic studies, appear to be appropriate markers of the taxonomic status of Salvia species (Gohari et al. 2011). It can be assumed that the variability observed in Salvia species arose due to adaptation, to alterations to the reproductive system in response to adverse environmental conditions and to recombination (Haque 1983; Wang et al. 2007; Baran et al. 2008; Aktas et al. 2009). In addition, there is a correlation between the habitat in which the plant grows and the production of flavonoid compounds (Tomas-Barberan \& Wollenweber 1990). The high incidence of flavonoid compounds in the Salvia genus largely consisted of few taxonomic units. To use flavonoid profiles more widely as genetic markers, they would have to be abundant and expedient, in order to identify the taxonomic position (Fairbbothers et al. 1975; Mika et al. 2005).

\section{Acknowledgments}

The author is grateful to the research deputy of Shahrekord University, which supported this study (Research Project no. 8812855).

\section{References}

Adzet, T.; Cai-Iigueral, S. \& Iglesias, J. 1988. A chromatographic survey of polyphenols from Salvia species. Biochemical Systematics and Ecology 16: 29-32.
Aktas, K.; Ozdemir, C.; Ozkan, M.; Akyol, Y. \& Baran, P. 2009. Morphologi$\mathrm{cal}$ and anatomical characteristics of Salvia tchihatcheffii endemic to Turkey. African Journal of Biotechnology 8: 4519-4528.

Amiri, H. 2007. Quantative and qualative changes of essential oil of Salvia bracteata Bank et Sol. in different growth stages. Daru 15: 79-82.

Anackov, G.; Bozin, B.; Zoric, L.; Vukov, D.; Mimica-Dukic, N.; Merkulov, L.; Igic, R.; Jovanovic, M. \& Boza, P. 2009. Chemical composition of essential oil and leaf anatomy of Salvia bertolonii Vis. and Salvia pratensis L. (Sect. Plethiosphace,Lamiaceae). Molecules 14: 1-9.

Bagcia, E.; Vuralb, M.; Dirmencic, T.; Bruehld, L. \& Aitzetmüllerd, K. 2004. Fatty acid and tocochromanol patterns of some Salvia L. species. Z. Naturforsch 59: 305-309.

Baikova, E.V. 1996. Floral morphology of some Salvia species as a reflection of its adaptation to pollinators and as basis for a generic system (in Russia). Byulleten Moskow Obsch Ispytat Prirody Biologii 4: 52-58.

Baran, P.; Ozdemir, C. \& Aktas, K. 2008. The morphological and anatomical properties of Salvia argentea L. in Turkey. Research Journal of Agriculture and Biological Sciences 4: 725-733.

Boissier, E. 1879. Flora Orientalis. 591-630. Geneva et Basilieae.

Ciesla, L.M. \& Waksmundzka-Hajnos, M. 2010. Application of thin layer chromatography for the quality control and screening the free radical scavenging activity of selected pharmaceutical preparations containing S. officinalis extract. Acta Poloniae Pharmaceutica 67: 481-485.

Fairbbothers, D.E.; Mabry, T.J.; Scogin, R.L. \& Turner, B.L. 1975. The bases of angiosperm phylogeny: chemotaxonomy. Annual Misouri Botanical Garden 92: 705-800.

Flamini, G.; Cioni, L.P.; Morelli, I. \& Bader, A. 2007. Essential oils of the aerial parts of three Salvia species from Jordan: Salvia lanigera, $S$. spinosa and S. syriaca. Food Chemistry 100: 732-735.

Gohari, A.R.; Ebrahimi, H.; Saeidnia, S.; Foruzani, M.; Ebrahimi, P. \& Ajani, Y. 2011. Flavones and flavone glycosides from Salvia macrosiphon Boiss. Iranian Journal of Pharmaceutical Research 10: 247-251.

Goren, A.C.; Kilic, T.; Dirmenci, T. \& Bilsel, G. 2006. Chemotaxonomic evaluation of Turkish species of Salvia: Fatty acid compositions of seed oils. Biochemical Systematic and Ecology 34: 160-164.

Gulen, H. \& Eris, A. 2004. Effect of heat stress in peroxidase activity and total protein content in strawberry plants. Plant Sciences 166: 379-744.

Habibvash, F.N. \& Rajamand, M.A. 2007. Anatomical observations on nutlets of some Salvia species from west Azerbaijan in Iran. Pakistan Journal of Biological Sciences 10: 3385-3389.

Habibvash, F.N.; Rajamand, M.A. \& Heidari, R. 2007. Study of some Salvia species native to west of Azerbaijan considering their phenolic compounds. Pakistan Journal of Nutrition 6: 443-446.

Haque, M.S. 1983. Phenotypic variability in foliar characters of some Salvia species. Proceeding of Indian natn Sciences Academy 49: 447-451.

Hedge, I.C. 1982a. Labiateae. Pp. 400-461. In: P.H. Davis (Ed.). Flora of Turkey. Edinburgh University Press, Edinburgh.

Hedge, I.C. 1982b. Labiateae. Pp. 403-476. In: Rechinger, K.H. (Ed). Flora Iranica. Akademische Druckund Verlagsanstalt, Graz, Austria.

Hedge, I.C. 1990. Labiateae. Pp. 193-217. In: Ali, S.I. \& Nasir, Y.J. (Eds). Flora of Pakistan. Pakistan, Department of Botany, University of Karachi.

Janicsak, G.; Veres, K.; Kakasy, A.Z. \& Mathe, I. 2006. Study of the oleanolic and ursolic acid contents of some species of the Lamiaceae. Biochemical Systematics and Ecology 34: 392-396.

Kahraman, A.; Celep, F. \& Dogan, M. 2009. A New Record for the Flora of Turkey: Salvia macrosiphon Boiss. (Labiatae). Turkish Journal of Botany 33: 53-55.

Kahraman, A. \& Dogan, M. 2010. Comparative study of Salvia limbata C.A. and S. palaestina Bentham (sect. Aethiopis Bentham, Labiatae) from East Anatolia, Turkey. Acta Botanica Croatica 69: 47-46.

Kharazian, N. \& Rahiminejad, M.R. 2008. Chemotaxonomy of wild diploid Triticum L. (Poaceae) species in Iran. International Journal of Botany 4: 260-268.

Kharazian. N. 2009. Taxonomy and morphology of Salvia spinosa in Iran. Taxonomy and Biosystematic Journal 1: 9-20.

Kharazian, N. \& Rahiminejad, M.R. 2009. Study of phenolic constituents of Triticum L. (Poaceae) species in Iran. Iranian Journal of Science \& Technology 33: 309-315. 
Kharazian, N. 2011. Karyotypic study of some Salvia (Lamiaceae) species from Iran. Journal of Applied Biological Sciences 5: 21-25.

Kharazian, N. 2012a. Taxonomy and morphology of Salvia atropatana in Iran. Journal of Sciences Tarbiat moallem University 11: 13-22

Kharazian, N. 2012b. Morphometry study of some Salvia L. species in Iran. Scientific Journal of Biological Sciences. 1: 126-137.

Khan, T.; Zahid, M.; Asim, M.; Shahzad, H.; Igbal, Z.; Choudhary, M.I. \& Ahmad, V.U. 2002. Pharmacological activities of crude acetone extract and purified constituents of Salvia Moorcraftiana Wall. Phytomedicine 9: 749-752.

Lu, Y. \& Foo, L.Y. 2000. Flavonoid and phenolic glycosides from Salvia officinalis. Phytochemistry 55: 263-267.

Lu, Y. \& Foo, L.Y. 2002. Polyphenolic in Salvia. Phytochemistry 59: $117-140$.

Maksimovic, M.; Vidic, D.; Milos, M.; Solic, M.E.; Abadzic, S. \& SiljakYakovlev, S. 2007. Effect of the environmental conditions on essential oil profile in two Dinaric Salvia species: S. brachyodon Vandas and S. officinalis L. Biochemical Systematics and Ecology 35: 473-478.

Markham, K.R. 1982. Pp. 10. Techniques of Flavonoid Identification. New York, Academic Press.

Mika, V.; Kuban, V.; Keljdus, B.; Odstrcilova, V. \& Nerusil, P. 2005. Phenolic compounds as chemical markers of low taxonomic levels in the Family Poaceae. Plant Soil Environment 51: 506-512.

Nakiboglu, M. 2002. The classification of the Salvia L. (Labiatae) species distributed in west Anatolia according to phenolic compounds. Turkish Journal of Botany 26: 103-108.

Nikolova, M.; Janicsak, G.; Genova, E. \& Mathe, I. 2006. Comparative analysis of external flavonoids of Bulgarian and Hungarian samples of Salvia species. Acta Botanica Hungarica 48: 361-367.

Ozdemir, C. \& Senel, G. 1999. The morphological, anatomical and karyological properties of Slavia sclerea L. Turkish Journal of Botany 23: 7-18.
Ozkan, M.; Aktas, K.; Ozdemir, C. \& Guren, G. 2009. Nutlet morphology and its taxonomic utility in Salvia (Lamiaceae: Mentheae) from Turkey. Acta Botanica Croatica 68: 105-115.

Pobedimova, E.G. 1954. Salvia L. pp: 178-260. In: B.K. Schischkin (ed). Flora of the USSR. Israel Prog. Sci.Transl, Jerusalem.

Sajjadi, S.E. \& Ghannadi, A. 2005. Essential oil of the Persian sage, Salvia rhytidea Benth. Acta pharmacology 55: 321-326.

Sajadi, S.; Shiran, B.; Kharazian, N.; Houshmand, S. \& Sorkheh K. 2010. Genetic diversity of Salvia species from Chaharmahal va Bakhtiari and Isfahan province using AFLP molecular markers. Journal of Horticulture Sciences 40: 79-88.

Salimpour, F.; Mazooji, A. \& Akhoondi, S. 2011. Chemotaxonomy of six Salvia species using essential oil composition markers. Journal of Medicinal Plants Research 5: 1795-1805.

Tomas-Barberan, F.A. \& Wollenweber, E. 1990. Flavonoid aglycones from the leaf surfaces of some Labiatae species. Plant Systematic and Evolution 173: 109-118.

Tosun, M.; Ercisli, S.; Sengul, M.; Ozer, H.; Polat, T. \& Ozturk, E. 2009. Antioxidant properties and total phenolic content of eight Salvia species from Turkey. Biological Resaerch 42: 175-181.

Valant-Vestachera, K.M.; Roitman, J.N. \& Wollenweber, E. 2003. Chemodiversity of exudate flavonoids in some members of the Lamiaceae. Biochemical Systematics and Ecology 31: 1279-1289.

Walker, J.B.; Sytsma, K.J.; Treutlein, J. \& Wink, M. 2004. Salvia (Lamiaceae) is not monophyletic: implication for the systematics, radiation, and ecological specialization of salvia and Tribe Mentheae. American Journal of Botany 91: 1115-1125.

Wang, B.Y.; Zhang, C.B.; Chen, X.L.; Li, R. \& Chen, Y.L. 2007. Analysis on genetic diversity of different Salvia miltiorrhiza geographical population in China. Zhongguo Zhong Yao Za Zhi 32: 1988-1991. 